\title{
DESERTIFICACIÓN Y DEGRADACIÓN AMBIENTAL: CONTEXTO Y PRÁCTICAS EN LA ESCUELA FAMÍLIA AGRÍCOLA DOM FRAGOSO - BRASIL
}

\author{
A. C. F. BONFIM ${ }^{1}$, L. P. BARBOSA ${ }^{3}$, D. D. MACHADO $^{1}$, M. C. DANTAS $S^{1,2}$ \\ ${ }^{1}$ Universidade Estadual do Ceará - UECE, Faculdade de Educação de Crateús - FAEC \\ ${ }^{3}$ Universidade Estadual do Ceará - UECE, Departamento de Pós-Graduação em Sociologia \\ campelodantas@hotmail.com²
}

Reclbido 10/03/2017 - Aceptado 26/04/2018

DOI: $10.15628 /$ holos.2018.5764

\section{RESUMEN}

El Estado de Ceará, en el noreste de Brasil, se destaca por tener áreas susceptibles a la desertificación. Dichas áreas reciben mayor atención con los procesos de deterioro en razón del avance de la degradación ambiental. La investigación busca averiguar el conocimiento de alumnos de la Escuela Familia Agrícola (EFA) Dom Fragoso, localizada en la ciudad de Independencia - Ceará, en el noreste brasileño, sobre el proceso actual de degradación del medio ambiente, causas y consecuencias. Para recompilar los datos, se aplicó un cuestionario con preguntas para 90 estudiantes de la EFA. Los entrevistados tienen una concientización de que la acción del ser humano constituye el factor principal que influencia el cambio climático, a partir de la extracción y explotación de los recursos naturales. Las principales acciones que creen ser importantes para la mitigación de los impactos del cambio climático son el desarrollo de educación ambiental y la movilización social. Se observa, con las indagaciones de los estudiantes, que la educación contextualizada, la interdisciplinariedad de los contenidos y prácticas de campo permiten una mejor comprensión, a partir del cambio en el contexto teórico y práctico.

PALAVRAS-CHAVE: acción humana; educación contextual; proceso de enseñanza y aprendizaje; región semiárida.

\section{DESERTIFICATION AND ENVIRONMENTAL DEGRADATION: CONTEXT AND PRACTICE IN SCHOOL FARM FAMILY}

\begin{abstract}
The state of Ceará stands out in northeastern Brazil for having areas susceptible to desertification. These areas receive greater attention in relation to the processes of deterioration due to advancement in environmental degradation. The research seeks to find out the knowledge of students of the Family Farm School (FFS) Dom Fragoso, located in the city of Independencia Ceará in northeastern Brazil, about the current process of degradation of the environment, causes and consequences. It was applied semi-structured questionnaire to 90 students of FFS. The interviewees
\end{abstract}

have a conception that the action of man is the main factor in the influence of climate change from the extraction and exploitation of natural resources. The main actions that they believe are important for this mitigation are the development of environmental education and social mobilization. It is observed with the students' research that the contextualized education, the interdisciplinarity of the contents and field practices, allow a better comprehension, starting from the change in the theoretical and practical context.

KEYWORDS: human action, contextual education, teaching-learning, semiarid region. 


\section{INTRODUCCIÓN}

En el Nordeste de Brasil están las llamadas Áreas Susceptibles a la Desertificación (ASD), las cuales reciben mayor atención, por parte de la comunidad científica y proyectos gubernamentales, con respeto a los procesos de deterioro, una vez que están en una fase avanzada del proceso de degradación ambiental. En estas zonas hay una reducción en la fertilidad del suelo y poco almacenamiento de agua, factores directamente relacionados con la pérdida de la biodiversidad que afectan el nivel de vida de las personas que viven en estas zonas, generando problemas sociales, económicos y políticos (Ministério do Meio Ambiente [MMA], 2004).

En esta perspectiva, el Estado de Ceará se caracteriza por una geografía donde el $90 \%$ de su territorio se cualifica como semiárido, y alrededor del $10 \%$ de la totalidad de su área está dañificada directamente por la suma de factores, incluyendo las actividades humanas, la susceptibilidad del suelo a la degradación y sus características climáticas. Alrededor del 55\% del área del Estado, en 150 municipios tienen grandes posibilidades de convertirse en desertificadas (Oliveira \& Coriolanus, 2010).

Las zonas más afectadas en Ceará se encuentran en el Centro-Norte, conocida como el Jaguaribe Medio y Sertão dos Inhamuns. En éstas, el inestabilidad ambiental provoca pérdidas de productividad y, por otro lado, las calificaciones de reciente salinización y la separación del suelo, la sedimentación de los cursos de agua y extenso proceso de deforestación, entre otros (Nascimento, Souza \& Cruz, 2007).

En investigaciones realizadas a partir de mapas basados en el índice de semiaridez y la susceptibilidad a la desertificación, Leite, Soareas y Martins. (1993), se observa que el proceso de degradación ambiental es considerado crítico en una superficie de $11,459.4 \mathrm{~km}^{2}$, o sea, $10 \%$ de Ceará. Tres grandes áreas resultaron evidentes para el índice de aridez (entre 0,30 y 0,40), que son: Irauçuba; Inhamuns y Sertão de Crateús, especialmente en los municipios de Arneiroz, Aiuaba, Tauá, Independencia, Crateús, Tamboril y Jaguaribe.

En este contexto, las instituciones, a ejemplo de las escuelas, tienen un papel clave en la mitigación de la degradación ambiental y en el freno al avance de la desertificación. En particular, destacamos las escuelas agrícolas. Estas instituciones se basan en una concepción de educación y de pedagogía con visas al fortalecimiento de la convivencia con el semiárido. En esa perspectiva, construyen la llamada Educación Contextualizada con el Semiárido, cuyo proceso educativo está mediado por la Pedagogía de la Alternancia, organizada por tiempos educativos: el tiempoescuela y el tiempo-comunidad. La intencionalidad pedagógica de ese modelo educativo consiste en desarrollar un aprendizaje mediado por un lenguaje contextualizado con el territorio y la cultura campesina.

El tiempo-escuela constituye un periodo de quince días en la escuela, con el objetivo de desarrollar el estudio teórico y técnico con los educandos, en que se profundiza la comprensión de los contenidos de cada área del conocimiento. En el tiempo-comunidad, los educandos desarrollarán actividades en sus comunidades en que se aplique, en el campo empírico, los contenidos teóricos y técnicos. Se trata de una experiencia educativo-pedagógica mediada por una dialéctica del conocimiento en sus dimensiones teórico-empíricas (Fraga \& Sousa, 2015; 
Barbosa, 2013). Las actividades y prácticas escolares desarrolladas en esta modalidad se destinan a la educación de calidad, el respeto, la producción, la formación social, la recuperación cultural y el desarrollo sostenible en su lugar de origen (Souza, 2008).

En esa perspectiva, el presente trabajo busca evaluar el conocimiento popular de los alumnos de la Escuela Familia Agrícola Dom Fragoso, en la ciudad de Independencia - CE acerca del creciente proceso de degradación del medio ambiente, causas y consecuencias en los últimos años.

\section{MATERIALES Y MÉTODOS}

\subsection{Escuela Família Agrícola Dom Fragoso (EFA)}

La Asociación Escuela Família Agrícola Dom Fragoso de Independencia (AEFAI) está ubicada en la comunidad de Santa Cruz, zona rural del municipio de Independencia, en el Centro Oeste del Estado de Ceará, en la región semiárida de Brasil. Constituye una asociación organizada por miembros de la sociedad civil, agricultores, facilitadores, familias de las comunidades, padres de alumnos, antiguos alumnos y otras entidades.

Esta Escuela Agrícola ofrece una doble formación: en nivel bachillerato y en el técnico agrícola. El proceso educativo tiene por acción pedagógica la formación de los jóvenes hijos de campesinos, que viven en el campo, y que asimilen un aprendizaje contextualizado con el semiárido. En ese sentido, el proceso pedagógico estimula una enseñanza que fortalezca la identidad con el campo y, al mismo tiempo, el aprendizaje de prácticas de producción adecuadas a la biodiversidad del semiárido (Fraga \& Sousa, 2015).

Por tal razón, se considera que el proceso educativo-pedagógico mediado por la EFA Dom Fragoso contribuye al desarrollo sostenible de las zonas rurales e integra las familias y las comunidades, lo que permite condiciones interdisciplinares indispensables para la transformación social, en un proceso formativo de carácter dialógico y dialéctico con la formación a nivel bachillerato y Técnico Agrícola.

Actualmente tiene un total de 98 alumnos, divididos en tres grupos de primero, segundo y tercero año, atendiendo a una demanda de más de 20 municipios de Ceará. Además, tiene un intercambio con el país de Cabo Verde, en el continente africano, con un estudiante. Desarrolla proyectos de educación para la convivencia con el semiárido, la valorización identitaria y cultural de los pueblos del campo, la conservación del medio ambiente, la producción agrícola, la generación de empleo y renta para las comunidades, así como con la captación de recursos junto a otras organizaciones de cooperación, con más de 27 instituciones nacionales e internacionales para mantener la escuela.

Conforme se presentó anteriormente, el proceso de enseñanza-aprendizaje utiliza la "Pedagogía de la Alternancia" como metodología. En esa perspectiva, se considera importante situar el lugar de inscripción de ese abordaje pedagógico en el proceso de construcción del conocimiento entre los educandos de la EFA Dom Fragoso. 


\subsection{La recolección de datos}

Para el desarrollo del presente estudio, se realizó un cuestionario semi-estructurado en la EFA Dom Fragoso, con el objetivo de obtener informaciones sobre las acciones individuales y en comunidad, la sociabilidad y el proceso de la coexistencia con el semiárido y su biodiversidad. Éstos constituyen los instrumentales que nortearon los análisis de la investigación, sobre todo en el abordaje de las prácticas de conservación ambiental, desarrollado dentro de la institución y las comunidades circunvecinas, en el ámbito del Bachillerato y Técnico Agrícola.

Los cuestionarios fueron aplicados a 90 alumnos en mayo de 2014, distribuidos de la siguiente manera: 32 alumnos de la clase de primer año, 25 en la clase de segundo año y 33 en el tercero año. Los resultados serán presentados con base en 05 ejes: 1 . Concepto y entendimiento sobre el proceso de desertificación; 2. Factores que desencadenan la desertificación; 3. Desarrollo de proyectos de conservación del medio ambiente; 4. Recursos naturales más degradados en las comunidades y 5. La acción humana y los factores naturales frente al cambio climático. Asimismo, los resultados serán refrendados con abordajes teórico-analíticos acerca de la temática en cuestión.

\section{RESULTADOS Y DISCUSIÓN}

\subsection{Concepto y entendimiento sobre el proceso de desertificación}

De acuerdo con las respuestas, se observó que, en su mayor parte, hay una comprensión del concepto de la desertificación y los procesos que los desencadenan. De los estudiantes entrevistados, la mayoría (59\%) demostró una compresión del concepto en base a la literatura correspondiente. Por otro lado, los entrevistados ejemplificaron las características de la desertificación y sus consecuencias, de acuerdo a su propia percepción del territorio, poniendo de relieve la acción del hombre y su relación de explotación con la naturaleza.

Existe un gran porcentaje de comprensión del concepto, con base en el conocimiento adquirido en la interdisciplinaridad, característica de la organización curricular de la EFA, en la articulación con la formación en técnico agrícola, así como en la gestión de sus recursos. Los estudiantes informaron que el proceso de desertificación está directamente relacionado con la acción del hombre, activo en la degradación ambiental de la deforestación, la quema, entre otros. Silva et al. (2009) afirman que el concepto de la desertificación, desde el principio, está directamente relacionado con la acción humana, en el que el manejo inadecuado de los recursos del medio ambiente, provoca un desequilibrio natural, que culmina con la pérdida de la fertilidad del suelo.

En algunas respuestas, se observó que hay un entendimiento de la tierra como el principal recurso a ser afectado por la gestión inadecuada, pero no es el único. Los mismos informan la infertilidad de la tierra como acción directa en la producción vegetal y consecuentemente animal, donde la escasez o ausencia de los recursos naturales no sólo causan problemas ambientales, sino también socioeconómicos, conforme se observa en las siguientes respuestas:

"Es el proceso de desertificación de una degradación ambiental en particular, y del suelo, este proceso, el suelo se queda estéril y se desprotege con altas tasas de erosión" A.J.S.F.

"Es el proceso de destrucción de la vida del suelo dejando infértiles y terminando con su constitución" D.F.L. 
"La desertificación es la falta de recursos naturales, causando varios impactos, tanto social, económico, como ambiental" A.R.L.S.

Se observó que $25 \%$ de los entrevistados comprenden los factores que desarrollan este proceso, pero confunden causas y consecuencias. Eso se debe a la no comprensión del proceso de desertificación como resultado de acciones humanas anteriores. Factores relacionados principalmente a aspectos socioeconómicos y culturales potencializan las acciones humanas que influyen en la degradación ambiental.

Alrededor de $16 \%$ de los estudiantes no entienden la desertificación como consecuencia de la devastación y la explotación de los seres humanos sobre los recursos naturales. Ellos creen que el proceso de desertificación es un solo factor, como la tala y desforestación de un área, o quemadas, que afectan sólo una característica de este sitio. Pedrancini, Corazza-Nune, Galuch, Moreira y Ribeiro (2007), señalan que existe un paralelismo entre la relación del alumno con los conceptos de un proceso de apropiación de la palabra y su significado verdadero. La relación entre los factores y acciones se entrelazan, confundiendo al estudiante en la verdadera definición, que puede ser el resultado de un pseudo-aprendizaje, es decir, que todavía no se logra aprehender de forma más precisa a los conceptos. Por lo tanto, existe así el dominio de la palabra, pero no de su concepto.

Sin embargo, la falta de entendimiento no se produce de forma aislada, no es sólo responsabilidad del estudiante. El uso de los libros de texto se destaca por Silva et al. (2009), como la fuente principal de la investigación, con influencia directa en el proceso de aprendizaje y el conocimiento del estudiante. Sin embargo, el material de enseñanza necesita de un enfoque más amplio de la conceptualización, la explicación de los factores, y para permitir el surgimiento de una reflexión crítica y mitigar los efectos de la desertificación. Como resultado, los estudiantes no reconocen como una población residente en zonas vulnerables y sin darse cuenta de los problemas que ocurren en su vida cotidiana.

\subsection{Los factores que desencadenan la desertificación}

Hubo una discrepancia entre los grupos al relacionar los factores de la desertificación, con la influencia del aprendizaje a partir de aspectos locales y de conocimiento cotidiano. Esta fue marcada de acuerdo con el grado de conocimiento de cada uno, a partir de los temas ya estudiados.

Los estudiantes del 10 año apuntan la acción de depredación de dominancia y explotación extrema del hombre sobre el medio ambiente. Se destacan las prácticas locales desarrolladas como, la deforestación, la quema, el uso de pesticidas y fertilizantes, que son el centro de la acción de estos jóvenes, al trabajar las prácticas de gestión adecuadas en la agricultura, que es la fuente económica principal. Según Araújo y Sousa (2011), las causas de la desertificación en el Nordeste brasileño están siempre relacionadas con la explotación de los recursos a través de prácticas incorrectas como sobrepastoreo y el cultivo excesivo, lo que daña el suelo, y modelos de desarrollo inmediatistas. 
Factores como el cambio climático, la contaminación y la extracción de minerales, desencadenadas por los principales procesos de urbanización e industrialización fueron observados en algunos informes, conforme las respuestas que se presentan a continuación:

\footnotetext{
"La necesidad del hombre a querer tener todo lo que ofrece la naturaleza. Y también quieren explorar y producir más "F.J.C.

"El progreso de la desertificación se inicia de grandes proyectos en los bosques, las presas y los bosques, un factor muy importante es también el calentamiento global, el clima no es favorable, y la explotación de minerales, y como tal, la forma en que manejamos nuestra agricultura R.N.S."

"El gran aumento de la agricultura convencional con máquinas y mayor uso de plaguicidas" J.L.S.
}

Para los grupos de 20 y 30 años, se observa más la relación explotación/degradación a nivel mundial. Se refieren a los procesos de industrialización, el crecimiento demográfico, el desarrollo del capital económico y el consumismo. También citan la transformación de las actividades del campo, con el uso de maquinaria pesada, los pesticidas y fertilizantes, recursos influyentes y la dependencia de los campesinos con las multinacionales. En esa perspectiva, estos estudiantes logran articular el concepto de desertificación a otros factores de orden económico, sobre todo aquellos relacionados a escalas más grandes sobre la industria, procesos químicos, y el agronegócio.

Para $46 \%$ de los encuestados, el factor principal es la agroindustria, con su producción a gran escala, lo que requiere la extracción vegetal, mineral y las quemadas. Otros factores, como el uso de leña, forraje y hierbas, minería de calcita y granito, entre otras rocas sedimentarias, la producción de ladrillos y tejas; en su mayoría ilegales, hecho con mano de obra agrícola, y exentos de una legislación de responsabilidad ambiental (Figura 1).

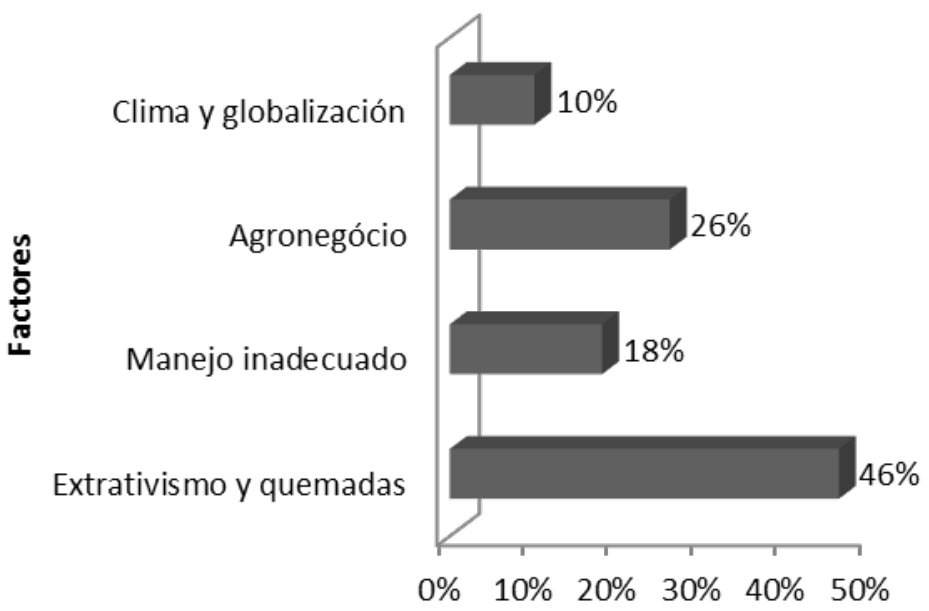

Figura 1: Relación de los factores que contribuyen al proceso de degradación/deforestación sobre el medio ambiente.

Para contraponerse a los efectos macroeconómicos en el proceso de desertificación, las y los educandos consideran fundamental que en su formación técnica desarrollen habilidades agrícolas que les permitan un manejo adecuado de los recursos naturales. Para el $18 \%$ de los 
estudiantes, el manejo inadecuado de los recursos naturales como el agua, el suelo y la vegetación, con la exploración excesiva y la contaminación, conducen a un proceso de degradación. Ellos hacen una correlación entre los recursos naturales y señalan que la ausencia de degradación de un recurso, cambia la calidad y disponibilidad del resto. Lima et al. (2011), enfatizan este aspecto al con el argumento de que el manejo adecuado de los recursos debe de ser sostenible y bien administrado, ya que es a partir de estos que se tienen beneficios económicos, sociales y ambientales.

Otro factor de desertificación descrito por $26 \%$ de los estudiantes fue la agropecuaria, con énfasis en la producción agrícola mecanizada, con el uso de pesticidas y monocultivos, procesos que fueron considerados inductores de la infertilidad y la contaminación del suelo. De igual manera la ganadería, con la creación de grandes animales y en grandes cantidades causa el pastoreo excesivo para el consumo y la exportación. En un estudio sobre la desertificación en el semiárido, Cavalcanti, Coutinho y Selva (2006), afirman que las actividades humanas son factores activos directamente en la erosión de los suelos, en la degradación de los recursos hídricos, de la vegetación, y casualmente en la perdida de la calidad de vida humana.

\subsection{Desarrollo de Proyectos de Conservación del Medio Ambiente}

Importante destacar que en el plan de formación y en el currículo de la EFA, cada educanda y educando desarrollará un proyecto, con el objetivo de implementar en lo concreto de la vida en sus comunidades, y que fortalezca la agricultura familiar. Constituye un momento crucial en la Pedagogía de la Alternancia, sobre todo en la construcción de un conocimiento dialéctico, en la articulación teoría-práctica, así como en el proceso de fortalecimiento de una pertenencia social, de los lazos de sociabilidad comunitaria, y de reafirmación de la identidad campesina.

Los estudiantes actúan en el desarrollo de trabajos y de sensibilización, de acuerdo con la necesidad de las comunidades asistidas. Cada proyecto de intervención tiene por característica la participación directa de la familia, o aún de la comunidad, con un enfoque basado en la conservación del medio ambiente. Estudio realizado por Araújo y Sousa (2011), demuestra que la contextualización de problemáticas ambientales locales en las clases puede ser utilizado como estrategia educativo-pedagógica para la comprensión y alerta ante la realidad de la comunidad.

A pesar de las dificultades encontradas, la escuela realiza una contribución efectiva de las prácticas educativas en las comunidades con el desarrollo de proyectos para la conservación del medio ambiente. Sin embargo, hay cuestiones que pueden afectar a las prácticas de éstos, como la falta de apoyo de la institución, las cuestiones burocráticas, la formación de profesionales y el salario.

Entre los estudiantes entrevistados, 64\% afirman que en sus comunidades existe algún proyecto de coexistencia y conservación del medio ambiente. Fueron citadas prácticas como la asistencia técnica a las familias, la reforestación, la oferta del agua, la producción de forraje, valoración cultural y social, además de la recogida selectiva. Acciones que buscan el desarrollo sostenible a partir de la generación de empleo y renta, con la producción agropecuaria y alianzas con algunas instituciones gubernamentales y Organizaciones No Gubernamentales (ONGs).

Las prácticas de campo y los proyectos de desarrollo van más allá de la aplicación de conocimientos, como lo demuestra Seniciato y Cavassan (2004), que afirman que éstas traen 
bienestar por el placer de vivir en un entorno diferente y agradable. También está la satisfacción con los conocimientos adquiridos en la práctica y el conocimiento de la naturaleza, en una relación activa que proporciona la recuperación y el acercamiento entre estudiantes y profesores.

Asimismo, 60\% de los estudiantes entrevistados afirmó que adoptan prácticas de gestión adecuadas desarrolladas por la asociación o por ellos mismos, en el ámbito interno de la familia, como el control de quemadas y la producción consciente. En este contexto, Fleury y Almeida (2007), señalan que los proyectos y prácticas de gestión son esenciales para la conservación del medio ambiente, para alimentar la relación entre la teoría y la práctica, y ayudar a vislumbrar una interacción sostenible entre las comunidades, sean las tradicionales o no.

Se observa que en las escuelas regulares, que tienen proyectos diferentes de la EFA Dom Fragoso, existe la necesidad de cambios en el proceso de enseñanza-aprendizaje, así como en las prácticas pedagógicas. Según Martins (2011), el cambio de paradigmas y el desarrollo de proyectos ambientales deben ser parte del Proyecto Político Pedagógico (PPP) de las escuelas, para contemplar las diferentes áreas de la educación y ser trabajado en todos los ámbitos.

Según Lucatto y Talamoni (2007), la enseñanza de metodologías de investigación-acciónparticipativa tienen mayores incidencias, tanto en el proceso de adquisición del conocimiento, como en el desarrollo de proyectos que beneficien directamente las familias y las comunidades. Con base en el análisis junto a los estudiantes, entre los principales resultados las prácticas desarrolladas en la EFA Dom Fragoso, está la participación de estudiantes, profesores y comunidades en proyectos de coexistencia y conservación del medio ambiente. Hay formación de grupos de estudio en su contexto para complementar la toma de decisiones por mejores proyectos de desarrollo, con la comprensión de los fenómenos y la búsqueda de la solución de los problemas encontrados en las comunidades.

\subsection{Los recursos naturales más degradados en las comunidades asistidas por la EFA Dom Fragoso}

Cuando se cuestionó con respecto a cuál recurso es más degradado en la comunidad, 35\% de los estudiantes afirma que es el suelo (Figura 2). Argumentan con base en las excavaciones y explosión de rocas para crear carreteras y la gestión agrícola inadecuada como factores de destrucción del suelo. En cuanto los desechos industriales y los vertederos a cielo abierto, existe la doble acción de los agentes que degradan el suelo y el agua, a partir del contacto con los ríos, la vegetación de ribera y las aguas subterráneas, que se cita como la degradación de los recursos agua en los municipios por $10 \%$ de los estudiantes.

La vegetación también fue citada como recurso degradado, donde $28 \%$ de los estudiantes describe las prácticas de deforestación (principalmente de los bosques nativos) para diversos fines, tales como leña, hierbas y la creación de espacios para la construcción civil. La madera se destaca como la principal fuente de energía de las comunidades rurales, debido a su fácil acceso. También hace hincapié en la cultura de la deforestación para la siembra agrícola, una práctica común en el semiárido.

Los resultados planteados por los estudiantes con respecto a los recursos naturales más degradados conllevan a la conclusión de que la eliminación de la vegetación nativa del semiárido 
afecta la biodiversidad local, además de interferir en las actividades relacionadas con el desarrollo social, económico y cultural. Es necesario que en estas áreas se adopten estrategias de lucha contra la destrucción de este ambiente, mejoren la calidad de vida de la población y que orienten a la utilización racional de los recursos naturales (Brasileiro, 2009).

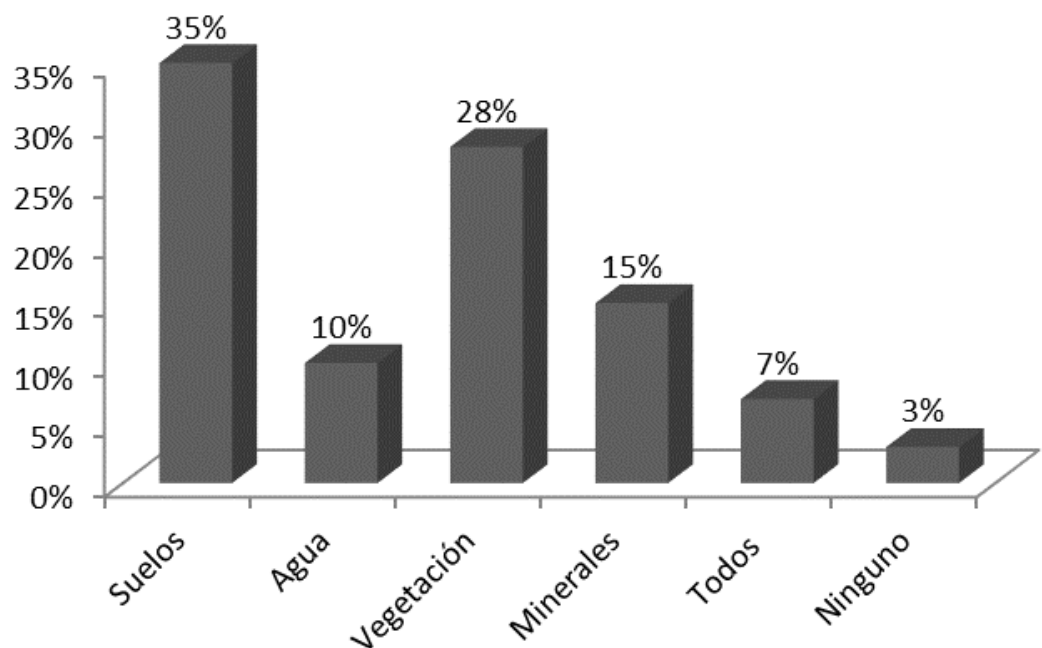

Figura 2: Descripción de los recursos más degradados en las comunidades atendidas por la EFA Dom Fragoso.

\subsection{La acción humana y los factores naturales frente al cambio climático en el planeta}

En esta pregunta se trató de exponer el conocimiento de los estudiantes acerca de los impactos causados por el hombre y los factores naturales que se hacen presentes desde el comienzo del planeta Tierra. También trató de hacer un paralelo de estas relaciones en la búsqueda de identificar cuál de ellas tiene una mayor influencia en el cambio climático actual.

De los entrevistados, $90 \%$ dijeran que era la acción del hombre el principal factor de influencia del cambio climático, de la extracción y explotación de recursos naturales. Mientras que sólo el $3 \%$ indican los factores climáticos y una cuota del 7\%, ambos. Este resultado demuestra que los estudiantes tienen una concepción adecuada de la degradación y la desertificación, cuanto la relación de devastación del hombre. Esto se refuerza a partir de las prácticas de conservación y preservación, una vez que aprenden cómo mantenerse de forma sostenible, sin degradar los recursos existentes, a partir del conocimiento de las zonas muy degradadas que trabajan.

Es menester destacar que el conocimiento de los estudiantes con respecto a los factores desencadenadores del cambio climático refrenda otros análisis relacionados a la fuerte influencia de la acción humana sobre los factores naturales, a propósito de la ocupación de zonas no aptas para la vivienda. En esa dirección, Cavalcanti et al. (2006), resaltan la relación entre los desastres ambientales y la devastación ambiental, al destacar que hay interferencia de carácter social, que requieren comprensión y conservación de las políticas públicas para mitigación de estos factores del cambio climático en la sociedad y el medio ambiente. 
Sobre el cambio climático y las acciones del hombre en los últimos años, hay muchas preguntas, ya que hay estudios que demuestran que la acción humana no afecta tan fácilmente sobre el cambio climático. Idea apoyado por Conti (2008) en trabajo sobre la desertificación.

\subsection{Acciones para combatir y mitigación de la degradación ambiental}

Según los estudiantes, hay muchas prácticas dirigidas hacia el combate y la minimización de los impactos ambientales. Fueron relatadas acciones como la recuperación del suelo, la concientización pública, cultivos consorciados, educación ambiental, fertilización verde y reforestación. Además de la integración de la agricultura familiar, la prevención, la conservación, la prohibición de la quema y la recogida selectiva.

En cuanto a las medidas adoptadas por estos estudiantes, 53\% describieran el desarrollo de la educación ambiental y la movilización social, para sensibilizar a las comunidades y fortalecer la lucha por la mejora de la vida. La participación activa de capturar en la búsqueda de inversiones para la agricultura familiar y el desarrollo sostenible del campo.

Pelegrini y Vlach (2011), describe que el abordaje de la educación ambiental en la escuela, la sensibilización y la intervención popular, se hace posible en este contexto, para dar forma a nuevos seres sociales, de la acción y la mitigación de los problemas ambientales y sociales, con el Estado. Traen también, la necesidad de incluir esto en la educación escolar de la escuela primaria como base para la comprensión de los procesos naturales, las crisis ambientales, acciones y reacciones antropogénicas, que influyen directamente en el nivel de vida de éstos.

Prácticas de agroecología, mencionados por 35\% de los estudiantes, desarrolladas en la institución y en la comunidad, son atractivos para los estudiantes porque son nuevas formas de trabajar la tierra y desarrollar el potencial de producción de cada área. Según Caporal y Costabeber (2002), el enfoque de la agroecología abarca muchos factores relacionados con áreas como la ecología, agronomía, economía, comunicación y otras. Las conexiones que redibujan la correcta gestión de los sistemas agrícolas para desarrollar de una manera sostenible en el tiempo.

Una minoría (12\%) de los estudiantes entrevistados dijo que la formación y el aprendizaje se necesitan más para que puedan ser actores clave en el desarrollo de prácticas de conservación de las comunidades de participar en eventos de la sociedad civil y de las comunidades rurales.

Los estudiantes entrevistados muestran que incluso fuera de la escuela, propagar y aplicar sus conocimientos, ya sea activa o pasivamente. Estos se insertan en forma participativa y activa de las comunidades, se relacionan y desarrollan proyectos con la población. De esta manera, aprenden y mejoran sus conocimientos y prácticas populares. Pensando esto con el apoyo de Jacobi (2003). Este nivel de conciencia diferente a los agricultores, como se demuestra por Oliveira, Barros y Silva (2012), que a pesar de estar sensibilizado a la degradación del medio ambiente, no utilizan buenas prácticas para el manejo del suelo.

Con respecto a la agroecología, Brasileiro (2009) afirma que la ciencia trata de combinar el conocimiento de los agricultores con el conocimiento técnico, a través de una relación más estrecha entre los conceptos y métodos de esta área y las prácticas de desarrollo sostenible. Permite al agricultor una idea de su relación con el medio ambiente. Este proceso valora los 
pequeños agricultores mediante el mantenimiento de los recursos naturales y de la adición de valor a la labor de los agricultores familiares.

\section{CONCLUSIONES}

La investigación reveló que la mayor parte de los alumnos de la Escuela de Familia Agrícola Dom Fragoso comprende el concepto de desertificación y los procesos que la desencadenan, en base a la literatura y en el conocimiento adquirido en la interdisciplinariedad característica de la organización curricular de la escuela. Es menester destacar que estos factores fueron identificados de forma estratificada, de acuerdo con el grado de conocimiento adquirido en los años ya estudiados, en que alumnos de los años iniciales apuntan la acción de explotación del hombre sobre el medio, destacándose prácticas locales de manejo inadecuado. Para los años finales se percibe la representación de la relación explotación / degradación a escala global, en que se relacionan los procesos de industrialización, crecimiento poblacional, desarrollo económico del capital y el consumismo.

Con respecto a ellos, se observa que tienen conocimiento sobre la degradación de los recursos naturales citando elementos de doble acción, como la contaminación de suelos, ríos y sábanas freáticas. El énfasis fue dado, en su mayor escala, a la degradación de suelos y vegetación. Los entrevistados tienen una concientización de que el factor principal de influencia del cambio climático, a partir de la extracción y explotación de los recursos naturales, es la acción del hombre. Por lo tanto, observase una concepción adecuada sobre degradación y desertificación, proceso fortalecido a partir de prácticas de conservación y preservación, en que aprenden a mantenerse sostenidamente, sin degradar los recursos existentes en áreas degradadas. Considerase fundamental dominar conceptos sobre la problemática de la infertilidad del suelo que afecta directamente a la producción vegetal y animal, lo que conlleva al desarrollo de problemas ambientales y socioeconómicos.

Las principales acciones que creen ser importantes para esta mitigación son el desarrollo de educación ambiental y movilización social, para fortalecer las luchas por la mejoría de la vida y búsqueda activa por incentivos para el desarrollo sustentable del campo, prácticas agroecológicas, que son muy estimulantes, así como la capacitación y el aprendizaje.

Se observa con las indagaciones de los estudiantes que la educación contextualizada, la interdisciplinariedad de los contenidos y prácticas de campo, permiten una mejor comprensión, a partir del cambio en el contexto teórico y práctico. También activa la conservación, mejoramiento cultural y ambiental en la búsqueda del desarrollo sostenible y el crecimiento socio-económico. Las prácticas utilizadas en el proceso de enseñanza-aprendizaje y desarrollado proyectos operan en la formación de conceptos y la comprensión de los factores ambientales, así como, la influencia del hombre sobre el medio ambiente.

\section{REFERENCIAS}

Américo, J. H. P.; Carvalho, S. L.; Freitas Lima E. A. C. \& Araujo, C. A. M. (2007). Avaliação das condições Ambientais de algumas propriedades agrícolas: percepção ambiental de 
produtores rurais da região de Dobrada-SP. In: Congresso de Ecologia do Brasil, 8 Caxambu.

Araújo, C. S. F. \& Sousa, A. N. (2011). Estudo do processo de desertificação na Caatinga: uma proposta de Educação Ambiental. Ciência \& Educação, 17(4), 975-986.

Barbosa, L. P. (2013). Educación, movimientos sociales y Estado en América Latina: estudio analítico de las experiencias de resistencia contrahegemónica en Brasil y México. Tesis de Doctorado. Programa de Posgrado en Estudios Latinoamericanos. Universidad Nacional Autónoma de México.

Brasileiro, R. S. (2009). Alternativas de desenvolvimento sustentável no semiárido nordestino: da degradação à conservação. Scientia Plena, 5(5), 1-12.

Cavalcanti, E. R.; Coutinho, S. F. S. \& Selva, V. S. F. (2006). Desertificação e desastres naturais na região do semiárido brasileiro. Caderno de Estudos Sociais, 22(1), 19-31.

Conti, J. B. (2008). O Conceito de desertificação. Climatologia e Estudos da Paisagem, 3(2), 3952.

Caporal, F. R. \& Costabeber, J. A. (2002). Agroecologia: Enfoque científico e estratégico. Agroecologia e Desenvolvimento Rural Sustentável, 3(2), 13-16.

Fleury, L. \& Almeida, J. (2007). Populações tradicionais e conservação ambiental: uma contribuição da teoria social. Revista Brasileira de Agroecologia, 2(3), 3-19.

Fraga, R. C. Q. \& Sousa, J. R. F. (2015). Pedagogia da Alternância e prática educativa na Educação do Campo: experiência da Escola Família Agrícola Dom Fragoso no Ceará, Brasil. Revista CISMA, (6), 3ㅇano.

Jacobi, P. (2003). Educação Ambiental, Cidadania e Sustentabilidade. Cadernos de Pesquisa, n. 118 , p. $189-205$.

Leite, F. R. B.; Soares, A. M. L. \& Martins, M. L. R. (1993). Áreas Degradadas Susceptíveis aos Processos de Desertificação no Estado do Ceará - 2a aproximação. Simpósio Brasileiro de Sensoriamento Remoto, 8, Fortaleza, 156-161.

Lima, R. C. C.; Cavalcante, A. M. B. \& Marin, A. M. P. (2011). (Ed.). Desertificação e mudanças climáticas no semiárido brasileiro. Campina Grande: INSA-PB, 209p.

Lucatto, L. G. \& Talamoni, J. L. B. (2007). A Construção Coletiva Interdisciplinar em Educação Ambiental no Ensino Médio: A microbacia hidrográfica do Ribeirão dos Peixes como tema gerador. Ciência \& Educação, 13(3), 389-398.

MARTINS, J. S. (2011). Tecnologias Educacionais: metodologias para capacitação e difusão de informações a rede de ensino. In: Lima, R. C. C.; Cavalcante, A. M. B.; Marin, A. M. P. (Ed.). Desertificação e mudanças climáticas no semiárido brasileiro. Campina Grande: INSAPB, cap.5, p.91-111.

MATTOS, B. H. O. M. (2011). Educação do campo e práticas educativas de convivência com o semiárido: a Escola Família Agrícola Dom Fragoso. Fortaleza: Banco do Nordeste. 
MMA/BRASIL. Ministério do Meio Ambiente. (2004). Programa de Ação Nacional de Combate à Desertificação e Mitigação dos efeitos da Seca - PAN-BRASIL. 3a Ed. Brasileira. Brasília: MMA., 225p.

Nascimento, F. R.; Souza, M. J. N.; Cruz, M. L. B. (2007). Enfoque Geoambiental para o tratamento da Degradação/Desertificação no município de Sobral Nordeste do Brasil/Ceará. Ateliê Geográfico, 1(2), 49-70.

Nascimento, F. R. (2009). Identificação de áreas susceptíveis à desertificação em Bacia Intermitente Sazonal no Semi-árido Brasileiro. B. Goiano. Geogr., 29(2), 71-82.

Oliveira, R. R.; Barros, J. D. S. \& Silva, M. F. F. (2012). Desertificação e degradação ambiental: percepção dos agricultores no município de Cachoeira dos Índios/PB. Polêmica, 11(2).

Pedrancini, V. D.; Corazza-Nune, M. J.; Galuch, M. T. B.; Moreira, A. L. O. R. \& Ribeiro, A. C. (2007). Ensino e aprendizagem de biologia no ensino médio e a apropriação do saber científico e biotecnológico. Revista Electrónica de Enseñanza de Las Ciencias, 6(2), 299-309.

Pelegrini, D. F. \& Vlach, V. R. F. (2011). As múltiplas dimensões da Educação Ambiental: por uma ampliação da abordagem. Revista Sociedade \& Natureza, 23(2), 187-196.

Pereira, A. M. \& Almeida, M. I. S. (2011). Degradação ambiental e desertificação no semiárido mineiro: um estudo sobre o município de Espinosa (MG). Revista Geográfica de América Central, n. especial, 1-16.

Seniciato, T. \& Cavassan, O. (2004). Aulas de campo em ambientes naturais e aprendizagem em ciências: um estudo com alunos do ensino fundamental. Ciência \& Educação, 10(1), 133-147.

Silva, M. M.; Novaes, J. A. M. \& Parahyba Junior, R. A. (2009). Abordagem do tema desertificação nos livros didáticos de geografia e biologia no ensino médio: um estudo com alunos do ensino fundamental. Revista Okara, 3(1), 168-185.

Souza, B. I. (2008). Cariri Paraibano: do silêncio do lugar à desertificação. Porto Alegre: UFRGS, 198f. Tese Doutorado. 